Herrn

Dr. Fritz Britt

Vizedirektor Bundesamt für Gesundheit

3003 Bern

Basel, 2. März 2004

\title{
Sonographisches Hüftscreening bei Neugeborenen nach Graf
}

Lieber Herr Britt

Für Ihre ausführliche Antwort vom 14. Januar auf unsere Anfrage vom 19. Dezember danke ich Ihnen. Die NEK-CNE hat den Fall an ihrer Sitzung vom 4./5. Februar 2004 besprochen und Ihre Argumente sowie die Argumente der Kinderärzte diskutiert. Sie versteht, dass der präventive Nutzen des allgemeinen Hüftsonographie-Screenings, das erst provisorisch in den Leistungskatalog aufgenommen ist, auf der Grundlage wissenschaftlicher Evidenz einer Überprüfung unterzogen werden muss. Die Kommission möchte dazu aus ethischer Sicht aber folgendes zu bedenken geben:

Der Kostendruck darf nicht dazu führen, die Verletzung ethischer Grundsätze der medizinischen Forschung in Kauf zu nehmen. Die Zumutung eines vermeidbaren und erheblichen Risikos für die Neugeborenen, welche in einer solchen Studie in die Kontrollgruppe geraten, ist ethisch nicht zu rechtfertigen. Die Tatsache, dass es in der Schweiz Gebiete gibt, in denen das Screening bisher nicht eingeführt wurde - Romandie, Tessin -, macht eine absichtliche Unterlassung in der deutschen Schweiz, wo es faktisch eingeführt ist, nicht akzeptabler. Anders würde es allerdings aussehen, wenn sich retrospektiv zwischen diesen Populationen überhaupt keine Unterschiede feststellen liessen. Dies müsste man aber zuerst empirisch zeigen.

Unser Vorschlag ist, den präventiven Nutzen über die Resultate bei Neugeborenen anhand der gescreenten und nichtgescreenten Populationen retrospektiv zu prüfen. Sollten auch danach Unklarheiten bestehen bleiben, könnte man eine randomisierte Studie ins Auge fassen. Es wäre wünschenswert, dessen Protokoll zum gegebenen Zeitpunkt der Expertenkommission für genetische Untersuchungen beim Menschen gemäss GUMG, Art. 35, die sich auch mit Neugeborenenscreenings befassen muss, oder der NEK-CNE zur Stellungnahme vorzulegen.

Sie haben recht, dass die Durchführung eines Screenings mit schlechter Testgenauigkeit ebenfalls mit Risiken verbunden ist. Die Gefahr des Labellings und der Überbehandlung bei den falsch Positiven (breit Wickeln) liegen aber auf einer anderen qualitativen Ebene als das Inkaufnehmen von später nötig werdenden operativen Eingriffen bei unerkannten Diagnosen und scheinen uns deshalb - wie auch der Kostenfaktor - nicht vergleichbar zu sein.

Gerne stehen wir Ihnen für Gespräche zur Verfügung.

Mit herzlichen Grüssen, Ihr

Christoph Rehmann-Sutter, Präsident Nationale Ethikkommission im Bereich Humanmedizin NEK-CNE

Kopien

- Bundesrat Pascal Couchepin

- Direktor BAG, Prof. Thomas Zeltner

- Dr. med. Martin L. Schilt

- Präsident FMH, Prof. H. H. Brunner

- Eidgenössische Leistungskommission 